\title{
An Analytical Study on Boundary Layer Flow and Heat Transfer of Nanofluid Induced by a Non-Linearly Stretching Sheet
}

\author{
A. Malvandi ${ }^{1 \dagger}$, F. Hedayati ${ }^{2}$ and M. R. H. Nobari ${ }^{1}$ \\ ${ }^{1}$ Mechanical Engineering Department, Amirkabir University of Technology, Tehran, Iran \\ ${ }^{2}$ Department of Mechanical Engineering, Islamic Azad University, Sari Branch, Sari, Iran \\ †Corresponding Author Email: amirmalvandi@aut.ac.ir
}

(Received April 17, 2013; accepted September 9, 2013)

\begin{abstract}
Steady two-dimensional boundary layer flow of a nanofluid past a nonlinear stretching sheet is investigated analytically using the Homotopy Analysis Method (HAM). The employed model for nanofluid includes twocomponent four-equation non-homogeneous equilibrium model that incorporates the effects of Brownian motion $(\mathrm{Nb})$, thermophoresis $(\mathrm{Nt})$ and Lewis number ( $L e$ ) simultaneously. The basic partial boundary layer equations have been reduced to a two-point boundary value problem via the similarity variables. Analytical results are in best agreements with those existing in the literatures. The outcomes signify the decreasing trend of heat transfer rate with thermophoresis, Brownian motion and Lewis number. However, concentration rate has a sensitive behavior with parameters, especially the Brownian motion and thermophoresis parameters. Also, the weak points of numerical methods in such problems have been mentioned and the efficiency of HAM, as an alternative approach, in solving these kinds of nonlinear coupled problems has been shown.
\end{abstract}

Keywords: Nanofluid, Nonlinear stretching sheet, Homotopy analysis method, Thermophoresis, Brownian motion.

\section{NOMENCLATURE}

$\begin{array}{ll}C & \text { nanoparticle volume fraction } \\ D_{B} & \text { brownian diffusion coefficient } \\ D_{T} & \text { thermophoresis diffusion coefficient } \\ L e & \text { Lewis number } \\ n & \text { stretching parameter } \\ \mathrm{Nb} & \text { brownian motion parameter } \\ \mathrm{Nt} & \text { thermophoresis parameter } \\ \mathrm{Nu} & \text { Nusselt number } \\ \mathrm{Pr} & \text { Prandtl number } \\ \mathrm{Re} & \text { Reynolds number }\end{array}$

$\begin{array}{ll}\text { Sh } & \text { Sherwood number } \\ T & \text { temperature }(1 / \mathrm{K}) \\ w & \text { condition on the sheet } \\ \alpha_{m} & \text { thermal diffusivity }\left(\mathrm{m}^{2} / \mathrm{s}\right) \\ \eta & \text { similarity variable } \\ \lambda & \text { heat source } / \mathrm{sink} \\ \mu & \text { dynamic viscosity }(\mathrm{kg} / \mathrm{m} \cdot \mathrm{s}) \\ \tau & \text { parameter defined by }(\rho c)_{P} /(\rho c)_{f} \\ \phi & \text { rescaled nanoparticle volume fraction } \\ \infty & \text { ambient conditions }\end{array}$




\section{INTRODUCTION}

The study of the boundary layer flow over a stretching surface originated from the pioneering work of Crane (1970). Following Crane's study, thermal approach to this problem was investigated by Carragher and Crane (1982). They assumed temperature difference between the sheets and the ambient is proportional to a specific power of distance from the fixed point. Afterward, this concept extended to more practical cases such as viscoelastic fluid. (Rajagopal, Na et al. (1984)) and uniform heat flux boundary condition (Dutta et al. (1985)).

Later, Chen (1998) obtained the solution for laminar mixed convection flow adjacent to vertical, continuously stretching sheets. Flow and heat transfer in a second grade fluid over a stretching sheet was investigated by Vajravelu and Roper (1999). More recently, Vajravelu (2001) studied flow and heat transfer in a viscous fluid over a nonlinear stretching sheet by neglecting the viscous dissipation. Cortell (2007) carried out his study on the flow and heat transfer on a nonlinear stretching sheet at two different types of thermal boundary. Recently, Prasad et al. (2010) studied the mixed convection on heat transfer over a non-linear stretching surface with variable fluid properties. Later on, different concepts and applications of stretching sheets have been investigated in many fluid flow and heat transfer problems (Bachok et al. (2010), Chamkha and Ahmed (2011), Khan and Pop (2010), Vajravelu et al. (2011), Malvandi et al. (2012), Rana and Bhargava (2012), Singh et al. (2012), Malvandi (2013), Malvandi et al. (2013), Rasekh et al. (2013), Veerraju et al. (2012), Gangadhar (2012) and Mahapatra and Nandy (2013)).

In recent studies, scientists have realized that a more effective way for cooling is using nanofluids in which nanometer-sized particles are added into the working fluid. These tiny particles have high thermal conductivity, so the mixed fluids have better thermal properties (Lee et al. (1999)), Xuan and Li (2000), Das et al. (2003)). The material of these nanoscale particles is aluminum oxide $\left(\mathrm{Al}_{2} \mathrm{O}_{3}\right)$, copper $(\mathrm{Cu})$, copper oxide $(\mathrm{CuO})$, gold $(\mathrm{Au})$, silver $(\mathrm{Ag})$, etc., which are suspended in base fluids such as water, oil, acetone and ethylene glycol.

To obtain accurate solution of above-mentioned problems, numerical techniques have been developed for years but due to some restrictions (Liao (2004)), analytical approaches have been considered as an alternative by scientists. Perturbation technique is one of the most common methods in this field which are widely applied in science and engineering. The major disadvantageous of perturbation technique is that they strongly depend upon small/large physical parameters, so they cannot apply to strongly nonlinear problems. Hence, non-perturbation techniques such as Adomian decomposition method (Adomian, 1988) and Variational Iteration Method
(He, 1999) have been developed in order to avoid the dependency on small/large parameters. It must be noted that, these methods cannot ensure the convergence of series solution. On the other hand, The Homotopy Analysis Method (HAM) proposed by Liao (1992), is a general analytical approach to obtain series solutions of strongly nonlinear equations which can provide us a simple way to ensure the convergence of solutions series. In addition, we have great freedom to choose a proper base function to approximate a nonlinear problem. Therefore, the HAM is valid even for strongly nonlinear problems. Moreover, in contrast with numerical methods, it can be implemented with boundary condition at infinity. It is worth mentioning that one error is inevitable in numerical methods; because the physical domain is unbounded where as the computational domain has to be finite. In numerical methods, for all computations the far field boundary conditions denoted by $\eta_{\max }$ must be chosen appropriately with the aid of previous studies (Liao, 2004).

In this paper, the boundary layer flow and heat transfer of a nanofluid past a non-linearly stretching sheet has been studied analytically via Homotopy Analysis Method (HAM). The problem has recently been investigated numerically (Finite Element method) by Rana and Bhargava (2012) only for limited range of physical parameters by assuming $\eta_{\infty}=6$. It is not surprising that their results are limited to only special parameters that consistent with $\eta_{\infty} \leq 6$; however, current results are independent to the value of $\eta_{\infty}$ and cover a wide range of physical parameters. This method was used by many authors in the wide range of engineering problems (Joneidi et al. (2010), Ziabakhsh et al. (2010), Ziabakhsh et al. (2010), Hassani et al. (2011), Malvandi et al. (2014)). In Addition, the effects of non-dimensional parameters such as Prandtl number $\mathrm{Pr}$, Lewis number $L e$, Brownian motion number $\mathrm{Nb}$ and thermophoresis number $N t$ on the Nusselt and Sherwood numbers have been investigated in details.

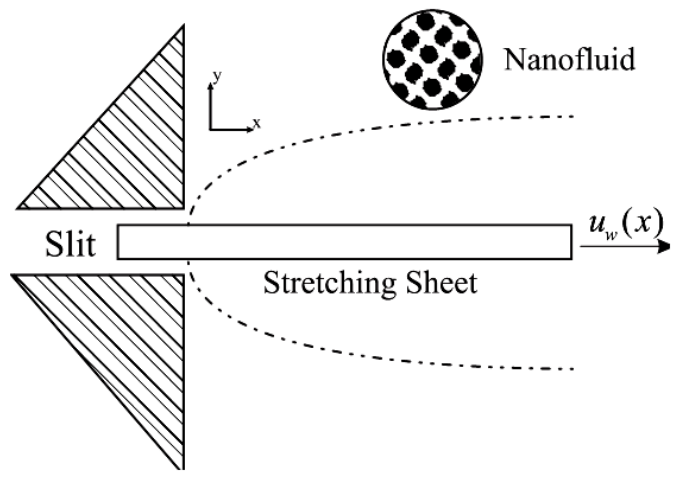

Fig. 1. Geometry of physical domain. 


\section{GOVERNING EQUATIONS}

Consider an incompressible steady viscous boundary layer flow of nanofluids flow over a flat plate moving as a function of $u_{w}=a x^{n}$, where $a$ is a constant, $n$ the nonlinear stretching parameter, and $x$ the coordinate measured along the stretching surface. . The geometry is shown in Fig.1. The wall temperature, $T_{w}$, is uniform and constant and is assumed greater than the free free stream temperature, $T_{\infty}$. Assuming the laminar boundary layer flow with negligible viscous dissipation, the continuity, momentum and energy

equations in the Cartesian coordinates can be expressed as (Rana and Bhargava (2012)).

$\frac{\partial u}{\partial x}+\frac{\partial v}{\partial y}=0$

$u \frac{\partial u}{\partial x}+v \frac{\partial u}{\partial y}=v\left(\frac{\partial^{2} u}{\partial x^{2}}+\frac{\partial^{2} u}{\partial y^{2}}\right)$

$u \frac{\partial T}{\partial x}+v \frac{\partial T}{\partial y}=\alpha_{m} \nabla^{2} T$

$+\tau\left[D_{B} \frac{\partial C}{\partial y} \frac{\partial T}{\partial y}+\frac{D_{T}}{T_{\infty}}\left(\frac{\partial T}{\partial y}\right)^{2}\right]$

$u \frac{\partial C}{\partial x}+v \frac{\partial C}{\partial y}=D_{B} \frac{\partial^{2} C}{\partial y^{2}}+\frac{D_{T}}{T_{\infty}} \frac{\partial^{2} T}{\partial y^{2}}$

where $C$ is the nanoparticle volume fraction and $\alpha_{m}=\frac{K_{m}}{(\rho c)_{f}}, \tau=\frac{(\rho c)_{P}}{(\rho c)_{f}}$.

The following boundary conditions are applied.

$$
\begin{aligned}
& \left\{\begin{array}{l}
u=u_{w}(x)=a x^{n}, v=0, T=T_{w}, \text { At } y=0 \\
C=C_{w}
\end{array}\right. \\
& \left\{\begin{array}{l}
u \rightarrow 0, T \rightarrow T_{\infty}, \\
C \rightarrow C_{\infty}
\end{array} \text { As } y \rightarrow \infty .\right.
\end{aligned}
$$

Here $u$ and $v$ are the velocity components along the $x$ and $y$ directions, respectively, $\rho_{f}$ the density of the base fluid, $\alpha_{m}$ the thermal diffusivity, $v$ the kinematic viscosity, $a$ a positive constant, $D_{B}$ the Brownian diffusion coefficient, $D_{T}$ the thermophoretic diffusion coefficient, $\tau=(\rho c)_{P} /(\rho c)_{f}$ the ratio between the effective heat capacity of the nanoparticle material and heat capacity of the fluid, $c$ the volumetric volume expansion coefficient, and $\rho_{p}$ the density of the particles. After using the similarity variables (Rana and Bhargava (2012)), Eqs. (1)to(4) may collapse into

$$
\begin{aligned}
& f^{\prime \prime \prime}+f f^{\prime \prime}-\left(\frac{2 n}{n+1}\right) f^{\prime 2}=0 \\
& \frac{1}{P r} \theta^{\prime \prime}+f \theta^{\prime}+N b \theta^{\prime} \phi^{\prime}+N t \theta^{\prime 2}=0 \\
& \phi^{\prime \prime}+\operatorname{Lef} \phi^{\prime}+\frac{N t}{N b} \theta^{\prime \prime}=0
\end{aligned}
$$

with the transformed boundary condition

$$
\begin{aligned}
& \text { At } \eta=0: \quad f=0, f^{\prime}=1, \theta=1, \phi=1 \\
& \text { As } \eta \rightarrow \infty: f^{\prime} \rightarrow 0, \theta \rightarrow 0, \phi \rightarrow 0
\end{aligned}
$$

where ' denotes differentiation with respect to $\eta$ and non-dimensional parameters are

$$
\begin{aligned}
& N b=\frac{(\rho c)_{p} D_{B}\left(C_{w}-C_{\infty}\right)}{(\rho c)_{f} v}, \operatorname{Pr}=\frac{v}{\alpha} \\
& N t=\frac{(\rho c)_{p} D_{T}\left(T_{w}-T_{\infty}\right)}{(\rho c)_{f} v T_{\infty}}, L e=\frac{v}{D_{B}}
\end{aligned}
$$

where $\mathrm{Pr}, \mathrm{Le}, \mathrm{Nb}, \mathrm{Nt}$ denote the Prandtl number, the Lewis number, the Brownian motion parameter and the thermophoresis parameter respectively. According to Bachok et al. (2012), the local Nusselt and Sherwood numbers can be defined as:

$$
\begin{aligned}
& N u=\frac{x q_{w}}{K\left(T_{w}-T_{\infty}\right)}, S h=\frac{x q_{m}}{D_{B}\left(C_{w}-C_{\infty}\right)}, \\
& C_{f}=\frac{\tau_{w}}{\rho u_{\infty}{ }^{2}}
\end{aligned}
$$

Here $\tau_{w}$ is the surface shear stress and $q_{w}, q_{m}$ are heat and mass flux at the surface respectively, and are defined as follows:

$$
\begin{aligned}
& \tau_{w}=\left.\mu\left(\frac{\partial u}{\partial y}\right)\right|_{y=0} \\
& q_{w}=-K\left(T_{w}-T_{\infty}\right) \sqrt{\frac{(n+1) a x^{n-1}}{2 v}} \theta^{\prime}(0) \\
& q_{m}=-D_{B}\left(C_{w}-C_{\infty}\right) \sqrt{\frac{(n+1) a x^{n-1}}{2 v}} \phi^{\prime}(0)
\end{aligned}
$$

It is worth mentioning that using dimensionless variables, the rate of heat and mass transfer and skin friction can be written as:

$\frac{N u}{\sqrt{R e_{x}}}=-\theta^{\prime}(0), \frac{S h}{\sqrt{R e_{x}}}=-\phi^{\prime}(0), \sqrt{2 R e_{x}} C_{f}=f^{\prime \prime}(0)$.

Like (Bachok et al. (2010)), in the present context $\mathrm{Nu} / \sqrt{R e_{x}}, \mathrm{Sh} / \sqrt{R e_{x}}$ and $\sqrt{2 R e_{x}} C_{f}$ are referred as the reduced Nusselt number, reduced Sherwood number and reduce skin friction coefficient which are represented by 
$-\theta^{\prime}(0),-\phi^{\prime}(0)$ and $f^{\prime \prime}(0)$, respectively. Due to unavailability of an exact solution for the case $n \neq 1$, the solution of highly nonlinear Eqs. (6) to (8) with Homotopy Analysis Method (HAM) as a semi-analytical technique will be discussed in the next section.

\section{SEMI-ANALYTICAL SOLUTION}

For HAM solutions, the appropriate initial guesses can be chosen as:

$$
f_{0}(\eta)=1-e^{-\eta}, \theta_{0}(\eta)=e^{-\eta}, \phi_{0}(\eta)=e^{-\eta} .
$$

and auxiliary linear operators.

$$
\begin{aligned}
& L(f)=f^{\prime \prime \prime}-f^{\prime}, \quad L\left(C_{1}+C_{2} e^{\eta}+C_{3} e^{-\eta}\right) \\
& L(\theta)=\theta^{\prime \prime}-\theta, \quad L\left(C_{4} e^{\eta}+C_{5} e^{-\eta}\right) \\
& L(\phi)=\phi^{\prime \prime}-\phi, \quad L\left(C_{6} e^{\eta}+C_{7} e^{-\eta}\right) .
\end{aligned}
$$

Where $c_{i}(i=1-7)$ are constants and $p \in[0,1]$ denotes the embedding parameter and $h$ indicate the non-zero auxiliary parameters. So, the zerothorder deformation problems are constructed as follows

$$
\begin{aligned}
& (1-P) L_{1}\left[f(\eta ; p)-f_{0}(\eta)\right]=p h_{1} N_{1}[f, \phi, \theta] \\
& (1-P) L_{2}\left[\theta(\eta ; p)-\theta_{0}(\eta)\right]=p h_{2} N_{2}[f, \phi, \theta] \\
& (1-P) L_{3}\left[\phi(\eta ; p)-\phi_{0}(\eta)\right]=p h_{3} N_{3}[f, \phi, \theta]
\end{aligned}
$$

subject to the following boundary conditions

$$
\begin{aligned}
& f(0, p)=0 \quad f^{\prime}(0, p)=0 \quad f^{\prime}(\infty, p)=1 \\
& \theta(0, p)=1 \quad \theta(\infty, p)=0 \\
& \phi(0, p)=1 \quad \phi(\infty, p)=0
\end{aligned}
$$

where

$$
\begin{aligned}
& N_{1}[f, \phi, \theta]=\frac{\partial^{3} f(\eta, p)}{\partial \eta^{3}}+f(\eta, p) \frac{\partial^{2} f(\eta, p)}{\partial \eta^{2}} \\
& -\left(\frac{2 n}{n+1}\right)\left(\frac{\partial f(\eta, p)}{\partial \eta}\right)^{2} \\
& N_{2}[f, \phi, \theta]=\frac{1}{P r} \frac{\partial^{2} \theta(\eta, p)}{\partial \eta^{2}}+f(\eta, p) \frac{\partial \theta(\eta, p)}{\partial \eta} \\
& +N b \frac{\partial \phi(\eta, p)}{\partial \eta} \frac{\partial \theta(\eta, p)}{\partial \eta}+N t\left(\frac{\partial \theta(\eta, p)}{\partial \eta}\right)^{2} \\
& N_{3}[f, \phi, \theta]=\frac{\partial^{2} \phi(\eta, p)}{\partial \eta^{2}}+L e\left(\frac{\partial \phi(\eta, p)}{\partial \eta}\right) f(\eta, p) \\
& +\frac{N t}{N b}\left(\frac{\partial^{2} \theta(\eta, p)}{\partial \eta^{2}}\right)
\end{aligned}
$$

According to the Taylor series with respect to $p$, the mth-order deformation equations may be achieved

$$
\begin{aligned}
& L_{1}\left[f_{m}(\tau)-\chi_{m} f_{m-1}(\tau)\right]=h_{1} R_{m}^{f}(\eta) \\
& L_{2}\left[\theta_{m}(\tau)-\chi_{m} \theta_{m-1}(\tau)\right]=h_{2} R_{m}^{\theta}(\eta) \\
& L_{3}\left[\phi_{m}(\tau)-\chi_{m} \phi_{m-1}(\tau)\right]=h_{3} R_{m}^{\phi}(\eta)
\end{aligned}
$$

with

$f_{m}(\eta)=\frac{1}{m !} \frac{\partial^{m} f(\eta, p)}{\partial p^{m}}, \theta_{m}(\eta)=\frac{1}{m !} \frac{\partial^{m} \theta(\eta, p)}{\partial p^{m}}$

$\phi_{m}(\eta)=\frac{1}{m !} \frac{\partial^{m} \phi(\eta, p)}{\partial p^{m}}$

and the boundary conditions are

$$
\begin{aligned}
& f_{m}(0)=f^{\prime}{ }_{m}(0)=f^{\prime}{ }_{m}(\infty)=0 \\
& \theta_{m}(0)=\theta_{m}(\infty)=0 \\
& \phi_{m}(0)=\phi_{m}(\infty)=0
\end{aligned}
$$

where

$$
\begin{aligned}
& R_{m}^{f}=f^{\prime \prime \prime}{ }_{m-1}+\sum_{n=0}^{m-1} f_{m-1-n} f^{\prime \prime}-\left(\frac{2 n}{n+1}\right) \sum_{n=0}^{m-1} f^{\prime}{ }_{m-1-n} f_{n}^{\prime} \\
& R_{m}^{\theta}=\frac{1}{P r} \theta^{\prime \prime}{ }_{m-1}+\sum_{n=0}^{m-1} f_{m-1-n} \theta_{n}^{\prime}+N b \sum_{n=0}^{m-1} \phi^{\prime}{ }_{m-1-n} \theta_{n}^{\prime}+ \\
& N t \sum_{n=0}^{m-1} \theta^{\prime}{ }_{m-1-n} \theta_{n}^{\prime}
\end{aligned}
$$

and

$$
\chi_{m}= \begin{cases}0 & m \leq 1 \\ 1 & m>1\end{cases}
$$

which $h_{i}(i=1,2,3)$, is chosen in such a way that these three series are convergent at $p=1$.

Eq. (23) represents the system of non-homogeneous linear differential equations whose general solutions are the sum of complementary and particular solutions which can be expressed as:

$$
\begin{aligned}
& f_{m}(\eta)=f_{m}^{*}(\eta)+C_{1}^{m}+C_{2}^{m} \mathrm{e}^{\eta}+C_{3}^{m} \mathrm{e}^{-\eta} \\
& \theta_{m}(\eta)=\theta_{m}^{*}(\eta)+C_{4}^{m} \mathrm{e}^{\eta}+C_{5}^{m} \mathrm{e}^{-\eta} \\
& \phi_{m}(\eta)=\phi_{m}^{*}(\eta)+C_{6}^{m} \mathrm{e}^{\eta}+C_{7}^{m} \mathrm{e}^{-\eta}
\end{aligned}
$$

To determine the values of these unknown constants, the boundary conditions (22) may be applied. Invoking the boundary conditions for $f_{m}, \theta_{m}, \phi_{m}$ as $\eta \rightarrow \infty$, it can be obtained

$C_{2}^{m}=C_{4}^{m}=C_{6}^{m}=0$

Similarly using the conditions at $\eta=0$ in Eq. (25), it can be deduced to

$$
C_{1}^{m}=-C_{3}^{m}-f_{m}^{*}(0), C_{3}^{m}=\frac{\partial f_{m}^{*}(\eta)}{\partial \eta} \mid \eta=0
$$

$C_{5}^{m}=-\theta_{m}^{*}(0), \quad C_{7}^{m}=-\phi_{m}^{*}(0)$

Hence, the velocity $f(\eta)$, the temperature $\theta(\eta)$ and the concentration $\phi(\eta)$ can be obtained by 


$$
\begin{aligned}
& f(\eta)=f_{0}(\eta)+\sum_{m=1}^{\infty} f_{m}(\eta) \\
& \theta(\eta)=\theta_{0}(\eta)+\sum_{m=1}^{\infty} \theta_{m}(\eta) \\
& \phi(\eta)=\phi_{0}(\eta)+\sum_{m=1}^{\infty} \phi_{m}(\eta) \\
& E_{1}=\int_{0}^{\infty}\left(N\left[\sum_{i=0}^{m} F_{i}(\xi)\right]\right)^{2} d \xi \\
& E_{2}=\int_{0}^{\infty}\left(N\left[\sum_{i=0}^{m} \theta_{i}(\xi)\right]\right)^{2} d \xi \\
& E_{3}=\int_{0}^{\infty}\left(N\left[\sum_{i=0}^{m} \phi_{i}(\xi)\right]\right)^{2} d \xi
\end{aligned}
$$

The more quickly $E_{i}(i=1,2,3)$ decreases to zero, the faster the corresponding homotopy-series solution converges. So, at a proper order of approximation $m$, the corresponding optimal values of the convergence-control parameter will be obtained by the minimum values of $E_{i}$ which can be estimated as

$$
\frac{\partial E_{1}}{\partial c_{1}}=0, \frac{\partial E_{2}}{\partial c_{2}}=0, \frac{\partial E_{3}}{\partial c_{3}}=0 .
$$

It is worth mentioning that since Eq. (6) and (7) are coupled, in order to minimize $E_{2}$ and $E_{3}$ a least square technique should be applied.

\section{RESULTS AND DISCUSSION}

The system of Eqs. (6) to (8) and the boundary condition of Eq. (9) have been solved analytically via Homotopy Analysis Method (HAM). As pointed by Liao (2004), the convergence rate of approximation for the HAM solution strongly depends on the value of auxiliary parameter, $h_{i}(i=1,2,3)$. In order to seek the permissible values of $h_{1}, h_{2}$ and $h_{3}$ the functions of $f^{\prime \prime \prime}(0), \theta^{\prime}(0)$ and $\phi^{\prime}(0)$ are plotted at 20th-order of approximations. Figs. $2 \mathrm{a}$ and $2 \mathrm{~b}$ clearly depict the acceptable range for values of $h_{1}\left(-1.7<h_{1}<-0.3\right)$ and $h_{2}\left(-1.3<h_{2}, h_{3}<-0.2\right)$, respectively. The current calculations are based on the value of $h_{i=1,2,3}=-0.7$ for the most cases. The results of HAM for the reduced heat transfer and concentration rates, i.e. $-\theta^{\prime}(0)$ and $-\phi^{\prime}(0)$ are compared with those obtained numerically using Finite Element Method by Rana and Bhargava (2012) for different values of $n$ and $P r=2$ in Tables 1 and 2, respectively. As it is evident, the current results are in very good agreement with the results of Rana and Bhargava (2012). In view of ensuring the accuracy of upcoming analytical
In order to seek the permissible values of $h_{i}$, the considered functions have to be plotted for a specifc physical point at an appropriate order of approximations. Obtaining the suitable $h_{i}$, the averaged residual errors were defined to find the optimal convergence-control parameters.

results, averaged residual errors for $m=20$ have been obtained which was shown in Fig. 3. Also, the results from Runge-Kutta-Fehlberg method as a numerical scheme have been obtained which were shown by diamonds in all the figures.

It should be stated that Brownian motion originates from random drifting of suspended nanoparticles, on the other hand, thermophoresis is nanoparticle migration due to imposed temperature gradient across the fluid. Mentioned mechanisms is the two important slip mechanisms which appears as a result of nanoparticles' slip velocity to the base fluid. For hot surfaces, due to repelling the submicron sized particles, the thermophoresis tends to blow nanoparticle volume fraction boundary layer away from the surface. Also, owing to size scale of particles, Brownian motion has significant influence on the surronding liquids.

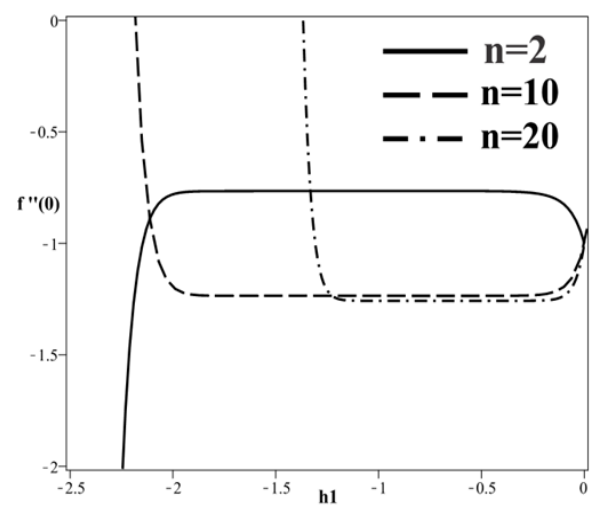

(a)

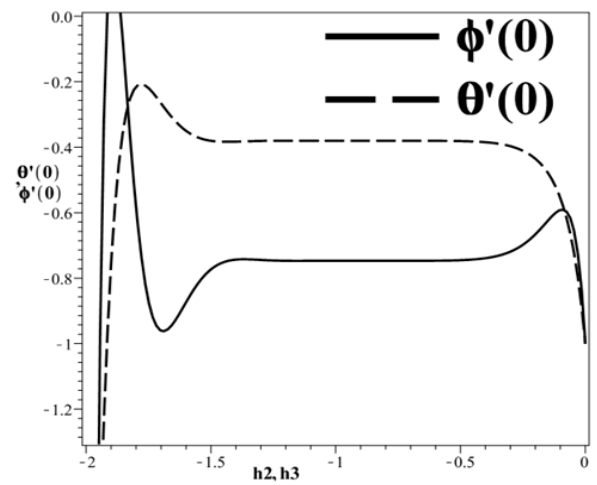

(b)

Fig. 2. $f^{\prime \prime}(0), \theta^{\prime}(0)$ and $\phi^{\prime}(0)$ plots for determining of $h_{1}, h_{2}$ and $h_{3}$ coefficients 


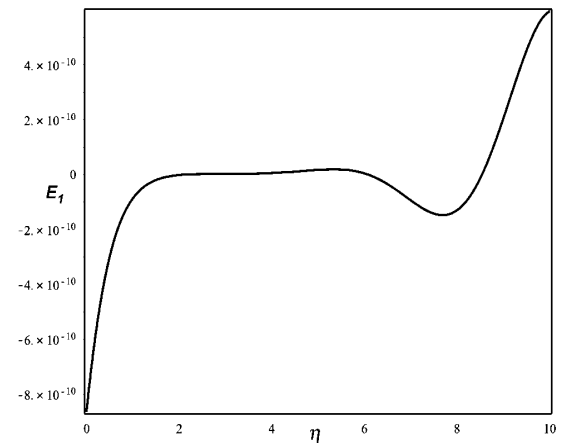

(a)

Table 1 Validation of results for reduced heat transfer rate for $P r=2$

\begin{tabular}{|c|c|c|c|c|}
\hline $\mathrm{n}$ & $\mathrm{Nt}$ & $\mathrm{Nb}$ & $\begin{array}{c}\text { Rana and } \\
\text { Bharghava } \\
(2012)\end{array}$ & HAM \\
\hline 0.2 & 0.1 & 0.5 & 0.5160 & 0.5157 \\
\cline { 3 - 5 } & & 2.5 & 0.0303 & 0.0302 \\
\cline { 3 - 5 } & 0.3 & 0.5 & 0.4533 & 0.4522 \\
& & & & 0.0263 \\
\cline { 3 - 5 } & & 2.5 & 0.0265 & 0.4854 \\
\hline 3 & 0.1 & 0.5 & 0.4864 & \\
\hline
\end{tabular}

Table 2 Validation of results for concentration rate for $\operatorname{Pr}=2$

\begin{tabular}{|c|c|c|c|c|}
\hline $\mathrm{n}$ & $\mathrm{Nt}$ & $\mathrm{Nb}$ & $\begin{array}{c}\text { Rana and } \\
\text { Bharghava } \\
(2012)\end{array}$ & HAM \\
\hline 0.2 & 0.1 & 0.5 & 0.9012 & 0.9003 \\
& & 2.5 & 0.9493 & 0.9497 \\
\cline { 3 - 5 } & \multirow{2}{*}{0.3} & 0.5 & 0.8395 & 0.8406 \\
\cline { 3 - 5 } & & 2.5 & 0.9571 & 0.9574 \\
\cline { 3 - 5 } & & & & 0.8450 \\
\hline 3 & 0.1 & 0.5 & 0.8445 & \\
\hline
\end{tabular}

Figures 4 and 5 show the effect of $N b$ and $N t$ in the transverse direction on the temperature and concentration profiles, repectively. As expected, temperature profile is similar to the conventional fluids. It can be seen that $\theta$ increases with $N b$ and this trends slightly vanishes for $N b>0.3$. Unlike $\theta$, the values of $\phi$, decreases by increasing $N b$.

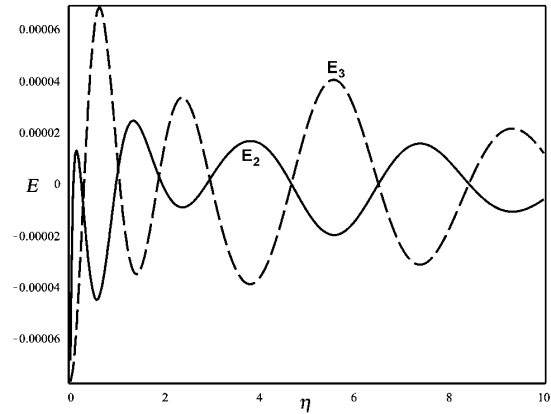

(b)

Fig. 3. Average residual errors which is defined by Eq. 29

Furthermore, it can be observed that at $N b=0.3$, the variational trend of $\phi$ changes. For lower

values of $N b<0.3$, concentration values inside the boundary layer reach a peak and then drop to its farfield value gradually. This trend extend the concentration profile which is more clear as $\mathrm{Nb}$ takes lowest value. Obviously in Fig. 5, an increase in the thermophoresis parameter $N t$ leads to increase in both temperature and nanoparticle concentration. Interesting behavior of concentration profile is that for $N t>1$, concentration rates change significantly which widen the concentration boundary layer noticeably.

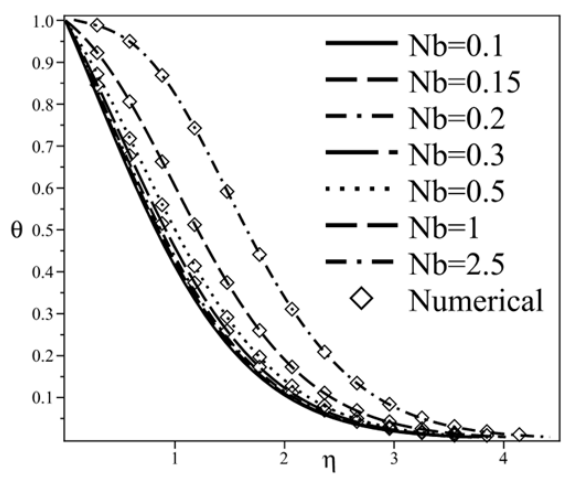

(a)

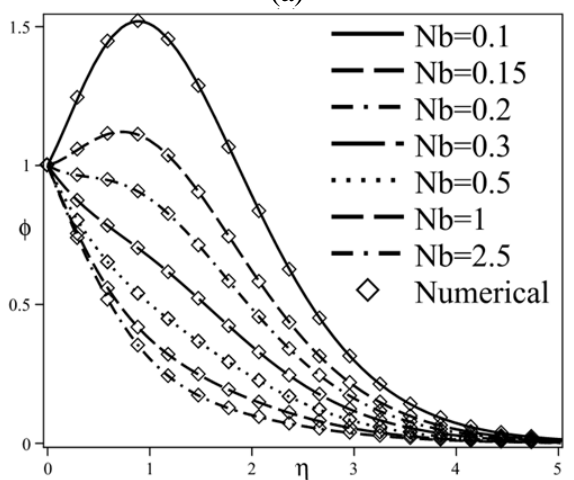

(b)

Fig. 4. Temperature and Concentration profiles for different values of $N b, n=L e=p r=2, N t=0.5$ 


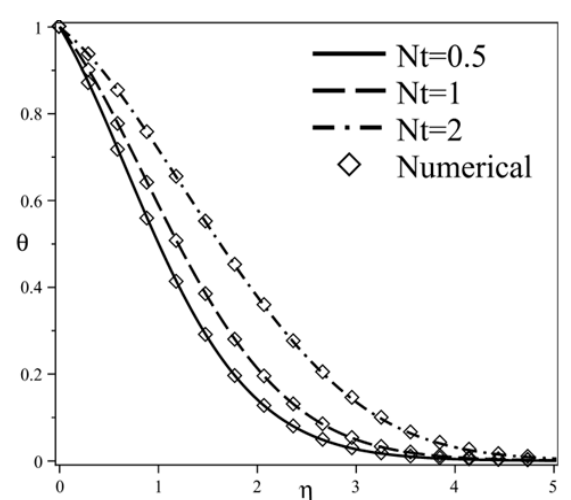

(a)

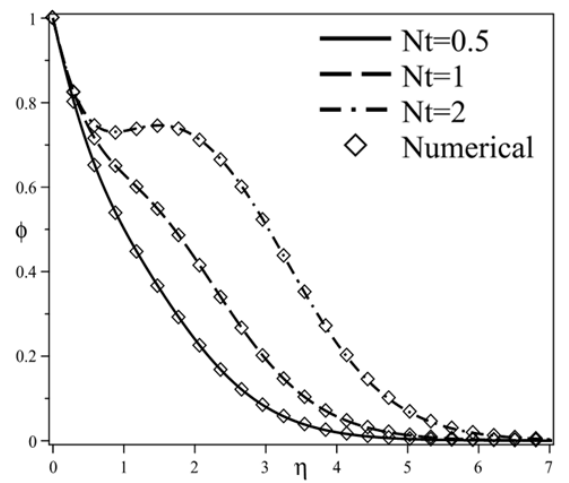

(b)

Fig. 5. Temperature and Concentration profiles for different values of $N t, n=L e=P r=2, N b=0.5$

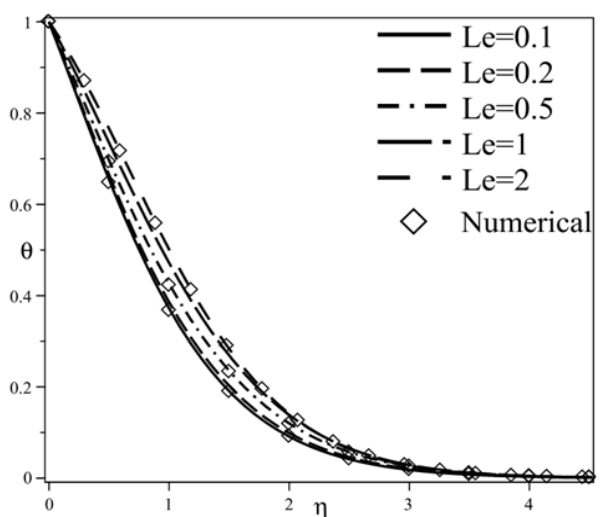

(a)

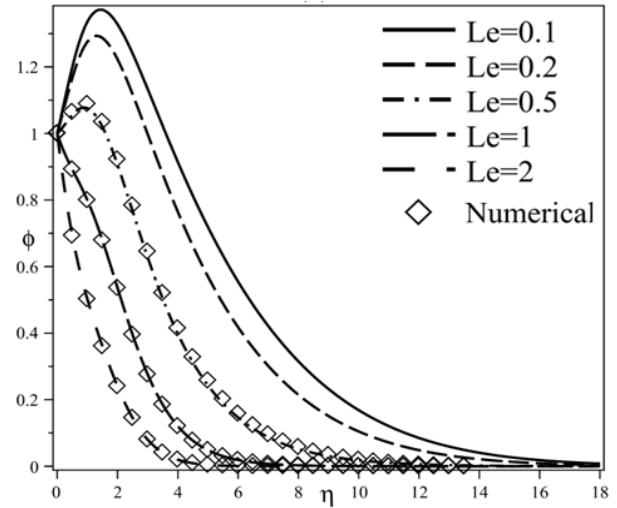

(b)

Fig. 6. Temperature and Concentration profiles for different values of

Le, $n=P r=2, N t=N b=0.5$
Figure 6 depicts the variations of temperature and concentration profiles with Lewis number. Lewis number defines the ratio of thermal diffusivity to mass diffusivity. It is used to characterize fluid flows where there is simultaneous hear and mass transfer by convection. It is clear that unlike the concentration profile, as Le increases the temperature profile decreases gently. It is worth mentioning that the concentration profile expands for the lower values of Lewis number. Moreover, for the lower values of Lewis number ( $L e<0.5$ ), the thickness of the concentration boundary layer increases $\left(\eta_{\infty}>10\right)$ which shows the assumed parameter in numerical studies $\left(\eta_{\infty}=6\right)$ does not cover the whole effective domain for this problem. Thus, the necessity of an efficient method that does not depend on $\eta_{\infty}$ became obvious. This suggests how applicable HAM is in solving these kinds of nonlinear coupled problems.

Figure 7 shows the effect of $L e$ on heat and concentration rate, respectively. In contrast to heat transfer rate, concentration rate takes an increasing trend with Le. Also, it can be seen that for $L e<1$, the concentration rate on the wall changes. i.e. opposite mass transfer occurs; this makes a noticeable rise in the concentration profile which continues with a downward trend to zero, See Fig. 6.

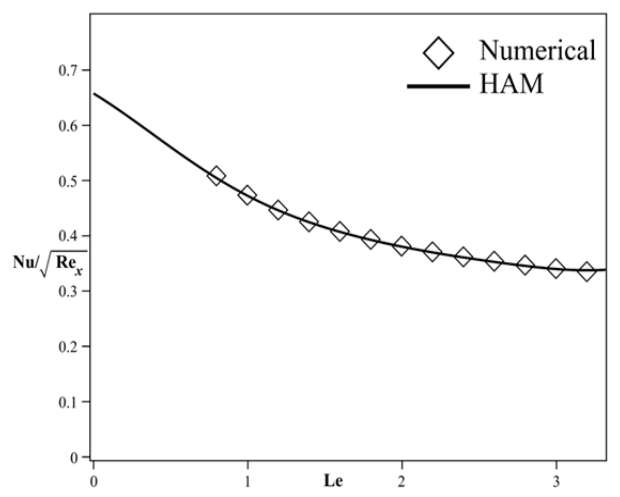

(a)

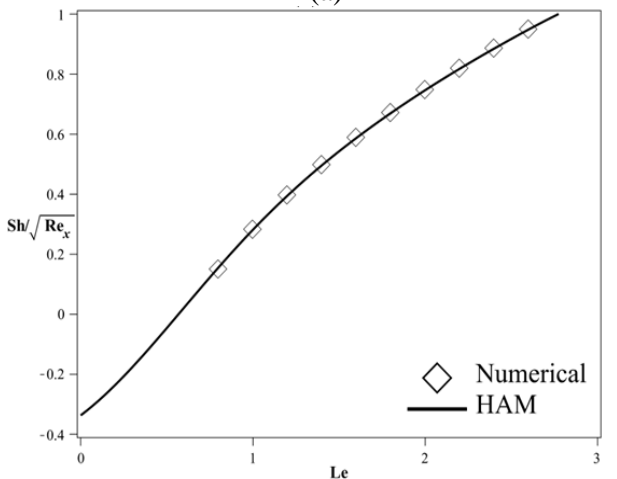

(b)

Fig. 7. Heat transfer (a) and concentration (b) rate for different values of $L e, N t=N b=0.5$ and $\operatorname{Pr}=n=2$. 


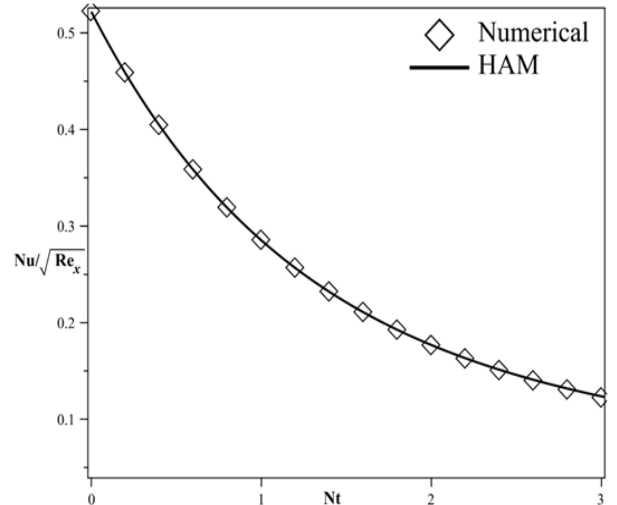

(a)

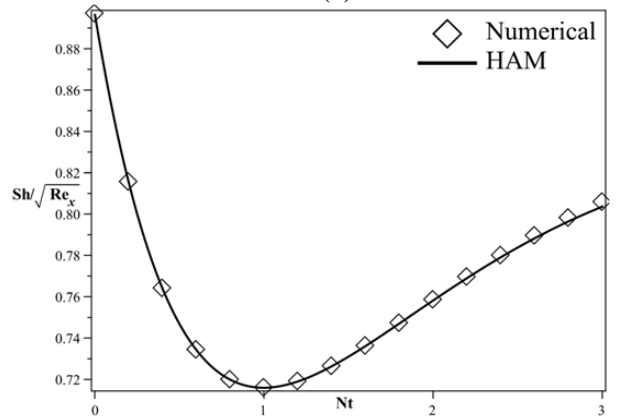

(b)

Fig. 8. Heat transfer (a) and concentration (b) rate for different values of

$$
N_{t}, L e=P r=n=2 \text { and } N b=0.5
$$

Figures 8 and 9 show the variation of heat and concentration rate versus thermophoresis and Brownian motion parameters. It can be observed that for $N t<1$, both values of $N u / \sqrt{R e_{x}}$ and $S h / \sqrt{R e_{x}}$ decrease; concentration rate takes an increasing trend after its minimal in $N t=1$. Based on Fig. 9, increasing in $N b$ leads to a marked increase in $S h / \sqrt{R e_{x}}$; however, $N u / \sqrt{R e_{x}}$ take a decreasing trend. What is more, there exist special values for $N b$ at which the values of $N u / \sqrt{R e_{x}}$ and $S h / \sqrt{R e_{x}}$ became independent of $N b$. These special values are $N b=3.5$ and $N b=1.5$ for heat and concentration rates, respectively. Also, it can be seen that for small value of $N b<0.5$, reverse concentration rate, i.e. negative Sherwood number occurs.

\section{Conclusions}

In this study, considering simultaneous effects of Brownian motion parameter, Lewis number and thermophoresis parameter, the boundary layer flow of nanofluids over a nonlinear stretching sheet has been studied. The transformed governing equations including continuity, momentum and energy have been solved analytically with Homotopy Analysis Method (HAM) and the obtained results are compared with the corresponding numerical solutions. Moreover, Effects of various parameters on temperature and concentration profiles are studied in detail. It was shown that for some values of parameters especially for lower values of Lewis number, due to increasing in the boundary layer thickness (physical domain) the numerical results do not cover the whole effective domain for these problems and hence the HAM is more suitable. Also, it was found that:

- When the Brownian motion parameter $N b$ increases, unlike temperature $\theta$, concentration $\phi$ decreases.

- An increase in the thermophoresis parameter, $N t$, leads to a rise in the values of temperature $\theta$ and concentration $\phi$ both.

- For the case $L e<1$ and $N b<0.5$ concentration rate may be reversed.

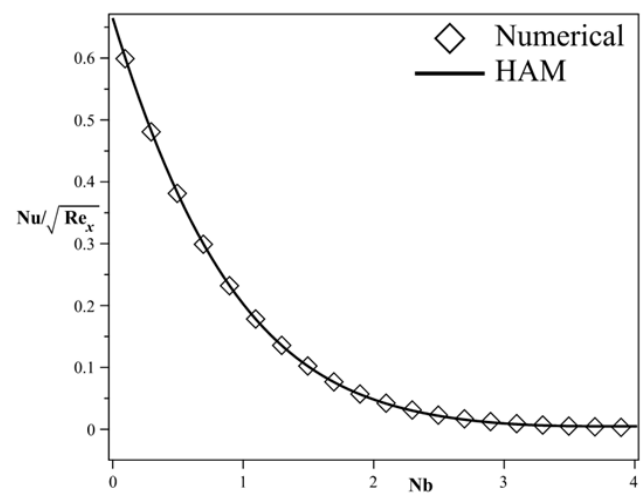

(a)

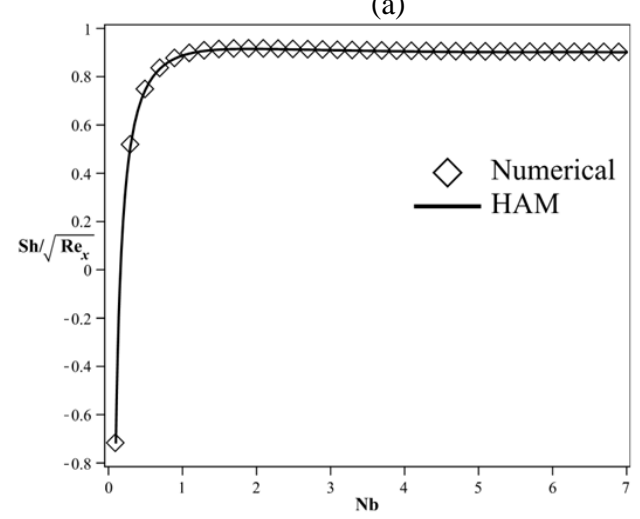

(b)

Fig. 9. Heat transfer (a) and concentration (b) rate for different values of $N b, L e=P r=n=2$ and $N t=0.5$

\section{REFERENCES}

Adomian, G. (1988). A review of the decomposition method in applied mathematics. Journal of Mathematical Analysis and Applications 135(2), 501-544. 
Bachok, N., A. Ishak and I. Pop (2010). Boundarylayer flow of nanofluids over a moving surface in a flowing fluid. International Journal of Thermal Sciences, 49(9), 1663-1668.

Carragher, P. and L. J. Crane (1982). Heat Transfer on a Continuous Stretching Sheet. ZAMM 62.

Chamkha, A. J., S. E. Ahmed (2011), Similarity Solution for Unsteady MHD Flow Near a Stagnation Point of a Three-Dimensional Porous Body with Heat and Mass Transfer, Heat Generation/Absorption and Chemical Reaction. Journal of Applied Fluid Mechanics, 4(3), 87-94.

Chen, C.H. (1998) Laminar mixed convection adjacent to vertical, continuously stretching sheets, Heat Mass Transfer, 33, 471-476.

Cortell, R. (2007). Viscous flow and heat transfer over a nonlinearly stretching sheet. Applied Mathematics and Computation, 184(2), 864873.

Crane, L. J. (1970). Flow past a stretching plate. $Z$ Angrew Math Phys., 21, 647.

Das, S. K., N. Putra and W. Roetzel (2003). Pool boiling characteristics of nano-fluids. International Journal of Heat and Mass Transfer, 46(5), 851-862.

Dutta, B. K., P. Roy and A. S. Gupta (1985). Temperature Field in Flow over a Stretching Sheet with Uniform Heat Flux. Int. Comm. Heat Mass Transfer, 12, 89.

Gangadhar, K. (2012), Soret and Dufour Effects on Hydro Magnetic Heat and Mass Transfer over a Vertical Plate with a Convective Surface Boundary Condition and Chemical Reaction. Journal of Applied Fluid Mechanics, 6(1), 95105.

Hassani, M., M. Mohammad Tabar, H. Nemati, G. Domairry and F. Noori (2011). An analytical solution for boundary layer flow of a nanofluid past a stretching sheet. International Journal of Thermal Sciences, 50(11), 2256-2263.

He, J.-H. (1999). Variational iteration method - a kind of non-linear analytical technique: some examples. International Journal of Non-Linear Mechanics, 34(4), 699-708.

Joneidi, A. A., G. Domairry and M. Babaelahi (2010). Analytical treatment of MHD free convective flow and mass transfer over a stretching sheet with chemical reaction. Journal of the Taiwan Institute of Chemical Engineers, 41(1), 35-43.

Khan, W. A. and I. Pop (2010). Boundary-layer flow of a nanofluid past a stretching sheet. International Journal of Heat and Mass
Transfer, 53(11 and 12), 2477-2483.

Lee, S., S. U. S. Choi and J. A. Eastman (1999) Measuring thermal conductivity of fluids containing oxidenanoparticles. ASME J Heat Transfer, 9, 280.

Liao, S. J. (1992). The proposed homotopy analysis technique for the solution of non-linear problems, Ph.D. Thesis, Shanghai Jiao Tong University.

Liao, S. J. (2004). On the Homotopy Analysis Method for Nonlinear Problems. Applied Mathematics and Computation, 147(2), 511.

Mahapatra, T. R., S. K. Nandy (2013), Momentum and Heat Transfer in MHD Axisymmetric Stagnation-Point Flow over a Shrinking Sheet. Journal of Applied Fluid Mechanics, 6(1), 121-129.

Malvandi, A. (2013). The Unsteady Flow of a Nanofluid in the Stagnation Point Region of a Time-dependent Rotating Sphere. Thermal Science Under Publication.

Malvandi, A., D. Ganji Domairry, F. Hedayati, M. Kaffash Hossein and M. Jamshidi (2012). Series solution of entropy generation toward an isothermal flat plate. Thermal Science, 16(5), 1289-1295.

Malvandi, A., F. Hedayati, D. D. Ganji and Y. Rostamiyan (2013). Unsteady boundary-layer flow of nanofluid past a permeable stretching/shrinking sheet with convective heat transfer. Journal of Mechanical Engineering Science Under Publication.

Malvandi, A., F. Hedayati and M. R. H. Nobari (2014). An HAM Analysis of Stagnation-point Flow of a Nanofluid over a Porous Stretching Sheet with Heat Generation. Journal of Applied Fluid Mechanics, 7(1).

Prasad, K. V., K. Vajravelu and P. S. Datti (2010). Mixed convection heat transfer over a nonlinear stretching surface with variable fluid properties. International Journal of NonLinear Mechanics, 45(3), 320-330.

Rajagopal, K. R., T. Y. Na and A. S. Gupta (1984). Flow of a Visco-Elastic Fluid over a Stretching Sheet. Rheol. Acta, 23.

Rana, P. and R. Bhargava (2012). Flow and heat transfer of a nanofluid over a nonlinearly stretching sheet: A numerical study. Communications in Nonlinear Science and Numerical Simulation, 17(1), 212-226.

Rasekh, A., A. Farzaneh-Gord, D. D. Ganji and S. Varedi (2013). Analytical solution for magnetohydrodynamic (MHD) stagnation point flow and heat transfer over a permeable stretching sheet with chemical reaction. 
A. Malvandi et al. / JAFM, Vol. 7, No. 2, pp. 375-384, 2014.

Journal of Theoretical and Applied Mechanics Accepted for publication.

Singh, P., D. Sinha, N. S. Tomer (2012), Oblique Stagnation-Point Darcy Flow towards a Stretching Sheet. Journal of Applied Fluid Mechanics, 5(3), 29-37.

Skobelev, B. I. , V. V. Struminskii (1977), Nonlinear perturbation development in twodimensional laminar flows. Journal of Applied Mathematics and Mechanics, 41, 5, 4

Vajravelu, K., T. Roper (1999), Flow and heat transfer in a second grade fluid over a stretching sheet, Int. J. Non-linear Mech., 34 (6), 1031-1036

Vajravelu, K. (2001). Viscous flow over a nonlinearly stretching sheet. Applied Mathematics and Computation, 124(3), 281288.

Vajravelu, K., K. V. Prasad, J. Lee, C. Lee, I. Pop and R. A. Van Gorder (2011). Convective heat transfer in the flow of viscous $\mathrm{Ag}$-water and $\mathrm{Cu}$-water nanofluids over a stretching surface.
International Journal of Thermal Sciences, 50(5), 843-851.

Veerraju, N., K. S. Srinivasa Babu, C. N. B. Rao (2012), Mixed Convection at a Vertical Plate in a Porous Medium with Magnetic Field and Variable Viscosity. Journal of Applied Fluid Mechanics, 5(4), 53-62.

Xuan, Y. and Q. Li (2000). Heat transfer enhancement of nanofluids. Int $J$ Heat Fluid Flow, 64, 58.

Ziabakhsh, Z., G. Domairry, H. Bararnia and H. Babazadeh (2010). Analytical solution of flow and diffusion of chemically reactive species over a nonlinearly stretching sheet immersed in a porous medium. Journal of the Taiwan Institute of Chemical Engineers, 41(1), 22-28.

Ziabakhsh, Z., G. Domairry, M. Mozaffari and M. Mahbobifar (2010). Analytical solution of heat transfer over an unsteady stretching permeable surface with prescribed wall temperature. Journal of the Taiwan Institute of Chemical Engineers, 41(2),169-17 